Check for updates

Cite this: Chem. Commun., 2020, 56,900

Received 29th October 2019

Accepted 11th December 2019

DOI: $10.1039 / \mathrm{c} 9 \mathrm{cc} 08438 \mathrm{j}$

rsc.li/chemcomm

\section{Synthesis of imides via palladium-catalyzed three- component coupling of aryl halides, isocyanides and carboxylic acids $\dagger$}

\author{
Bo Wang, $\ddagger$ Dan He, $\ddagger$ Beige Ren and Tuanli Yao (D) *
}

\begin{abstract}
A palladium-catalyzed three-component synthesis of acyclic imides from feedstock aryl halides, carboxylic acids and isocyanides through the intermediacy of isoimides has been developed. The key to the success of this approach was controlled isocyanide slow addition and organic/aqueous biphasic conditions. This transitionmetal-catalyzed approach features readily available starting materials, atom- and step-economy, good functional group compatibility and gram-scale synthetic capability. Utilization of this new method is illustrated in the late-stage functionalization of drugs Carprofen, Loxoprofen and Flurbiprofen. This strategy has also been successfully applied in the synthesis of cyclic imides including phthalimide, homophthalimide, and $2 \mathrm{H}$-2-benzazepine-1,3-dione derivatives.
\end{abstract}

Imides are present as a core structural motif in natural products and pharmaceutical agents. For example, natural products penimide $\mathrm{A},{ }^{1 a}$ berkeleyamide $\mathrm{C},{ }^{1 b}$ and pestalamides $\mathrm{A},{ }^{1 c}$ and approved drugs thalidomide, anircetam, ${ }^{1 d}$ and ethosuximide ${ }^{1 e}$ all contain an imide moiety (Scheme 1a). Imides also have a wide range of applications in agrochemicals ${ }^{2 a}$ and materials science. ${ }^{2 b}$ In addition, imides are versatile synthetic building blocks. ${ }^{3}$ The wide occurrence and high value of imides have driven the continuous development of new synthetic methods.

Mumm rearrangement of isoimides (Scheme $1 \mathrm{~b}$ ) is a traditional method for the synthesis of imides, ${ }^{4}$ in which the use of reactive reagents $\left(\mathrm{POCl}_{3}\right.$ and $\left.\mathrm{SOCl}_{2}\right)$ leads to limited functional group compatibility and creates significant chemical waste. Transitionmetal catalysed Mumm rearrangement has been developed recently. Unfortunately, these methods are limited to the synthesis of only specific imides such as $\mathrm{N}$-acyl propiolamides, ${ }^{5} \mathrm{~N}$-acetylindole-3-carboxamides ${ }^{6}$ and $\alpha$-amio imides. ${ }^{7}$ Furthermore, these methods require the use of pre-prepared carboxylic salts, which

Shaanxi Key Laboratory of Chemical Additives for Industry, College of Chemistry and Chemical Engineering, Shaanxi University of Science and Technology, Xi'an, 710021, China.E-mail: yaotuanli@sust.edu.cn

$\dagger$ Electronic supplementary information (ESI) available. CCDC 1962679. For ESI and crystallographic data in CIF or other electronic format see DOI: 10.1039/ c9cc08438j

\# These authors contributed equally.

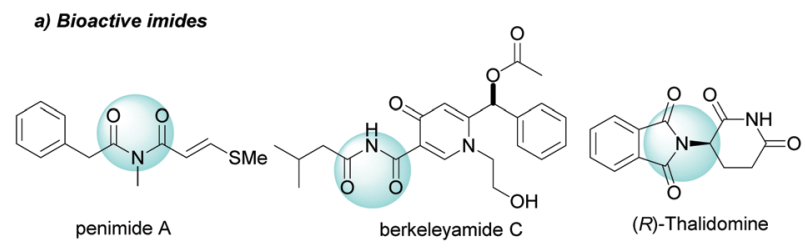

b) Tradational Mumm rearrangement

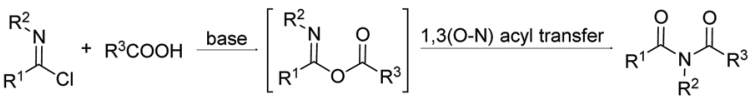

c) This work (Pd(0) catalyzed):

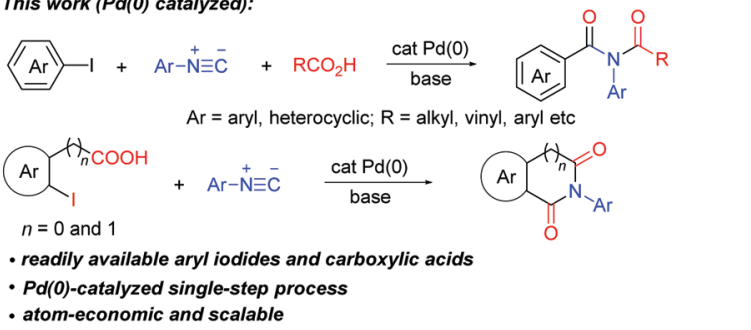

Scheme 1 Examples of biologically relevant imides, traditional Mumm rearrangement and palladium-catalyzed three-component synthesis of imides.

greatly limits the scope of applicable carboxylic acids. An ideal solution to overcome these limitations would be the development of a general and practical transition-metal-catalyzed process that utilizes feedstock chemicals such as aryl halides and carboxylic acids to arrive at acyclic imides. To the best of our knowledge, there is no example of catalytic imide synthesis employing aryl halides and carboxylic acids. Herein, we disclose the first palladium(0) catalyzed three-component coupling of aryl halides, isocyanides and carboxylic acids, which enables straightforward synthesis of a broad range of acyclic imides. This strategy has also been successfully applied in the synthesis of cyclic imides such as phthalimide, homophthalimide and 2-benzazepine-1,3-dione derivatives.

Our investigation of this Pd-catalyzed tandem process was commenced by surveying reaction conditions using isocyanide 
Table 1 Reaction optimization ${ }^{a}$

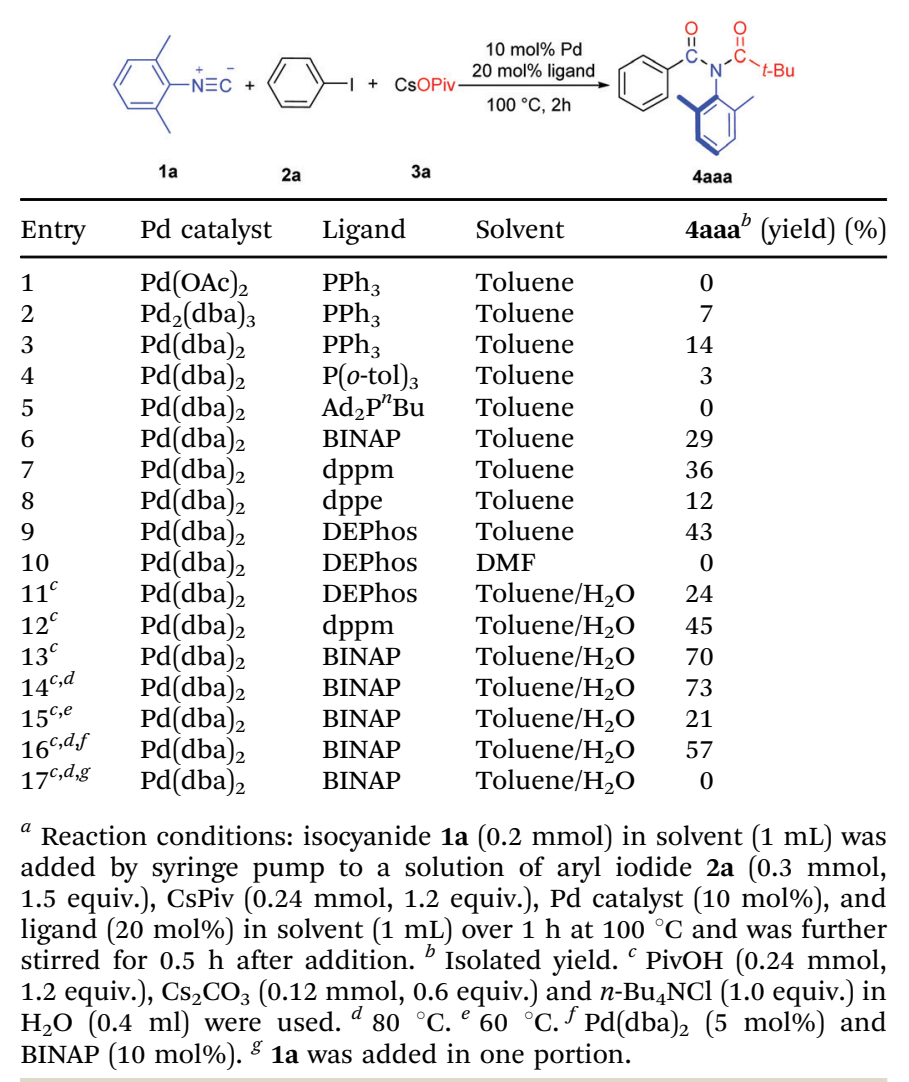

1a, aryl iodide 2a and cesium pivalate (3a) as the model. In the presence of $\mathrm{Pd}(\mathrm{OAc})_{2}(10 \mathrm{~mol} \%)$ and $\mathrm{PPh}_{3}$ (20 mol\%), no detectable imide $4 \mathbf{a}$ was produced (Table 1 , entry 1 ). To our delight, changing the palladium precatalyst to $\mathrm{Pd}_{2}(\mathrm{dba})_{3}$ or $\mathrm{Pd}(\mathrm{dba})_{2}$ afforded $\mathbf{4 a}$ in $7 \%$ and $14 \%$ isolated yields, respectively (Table 1, entries 2 and 3). The previously known indole product from isocyanide insertion/benzylic $\mathrm{C}\left(\mathrm{sp}^{3}\right)-\mathrm{H}$ activation was not observed. ${ }^{8}$ More sterically hindered $\mathrm{P}(o \text {-tol })_{3}$ and sterically demanding and electron-rich phosphine $\operatorname{Ad}_{2} \mathrm{P}^{n} \mathrm{Bu}$ are detrimental to the reaction (Table 1, entries 4 and 5). On the other hand, bidentate phosphines were more efficient and DEPhos afforded a 43\% yield of $4 a$ (Table 1, entries 6-9). Switching the solvent to more polar DMF was inferior (Table 1, entry 10). Since CsOPiv is very hydroscopic, an organic/ aqueous biphasic condition (toluene/water) using pivalic acid, $\mathrm{Cs}_{2} \mathrm{CO}_{3}$ and phase transfer reagent $n-\mathrm{Bu}_{4} \mathrm{NCl}$ was developed to facilitate operation and extend the reaction to more complex carboxylic acids (Table 1, entries 11-13). Remarkably, the isolated yield of $\mathbf{4 a}$ was increased to $70 \%$ upon using BINAP as the ligand (Table 1, entry 13) and no corresponding amide from reacting with water was observed. Decreasing the reaction temperature to $80{ }^{\circ} \mathrm{C}$ did not affect the result (Table 1, entry 14). Further decreasing the reaction temperature (Table 1, entry 15) or reducing the loading of the Pd catalyst (Table 1, entry 16) all led to lower yields of $\mathbf{4 a}$. It should be noted that slow addition of isocyanide 1a is the key to the success of the reaction. When adding $\mathbf{1 a}$ in one portion, no 4a was produced at all under otherwise identical conditions
Table 2 Scope of aryl halides ${ }^{a}$

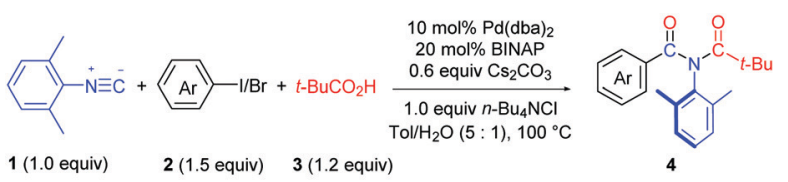

$\mathbf{1}$ (1.0 equiv) $\quad \mathbf{2}$ (1.5 equiv) $\mathbf{3}$ (1.2 equiv)
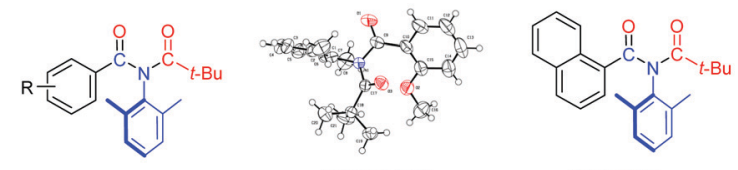

$\mathrm{R}=\mathrm{H}$

X-ray of 4 ada

4aia $(71 \%)$
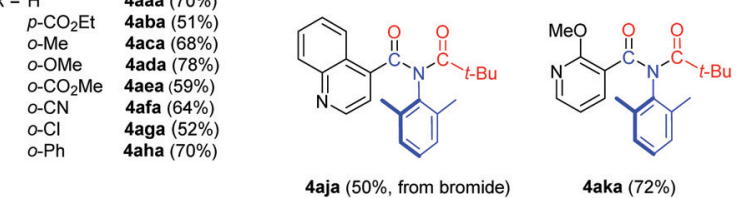

4aja (50\%, from bromide) 4aka (72\%)

${ }^{a}$ Performed on a $0.20 \mathrm{mmol}$ scale using the standard reaction conditions the same as Table 1, entry 13.

(compare Table 1, entries 14 and 17). We reasoned that slow addition of isocyanides would restrict the highly coordinating nature of isocyanides, ${ }^{9}$ which facilitated their insertion by the phenylpalladium intermediate and protected the catalyst from poisoning at the same time (see Scheme 3$){ }^{10}$

We first examined the substrate scope with respect to the aryl halides under the optimized reaction conditions (Table 1, entry 13), and the results are shown in Table 2 . Aryl iodides with either an electron-withdrawing or an electron-donating group worked smoothly in this chemistry (4aba-4aha). The structure of 4ada was confirmed by single crystal X-ray diffraction. ${ }^{11}$ It should be noted that 2-iodobiphenyl reacted chemoselectively, affording imide 4 aha in $70 \%$ yield without the formation of the previously reported fluorenone imine product. ${ }^{12}$ 1-Iodonaphthalene was also a

Table 3 Scope of isocyanides ${ }^{a}$
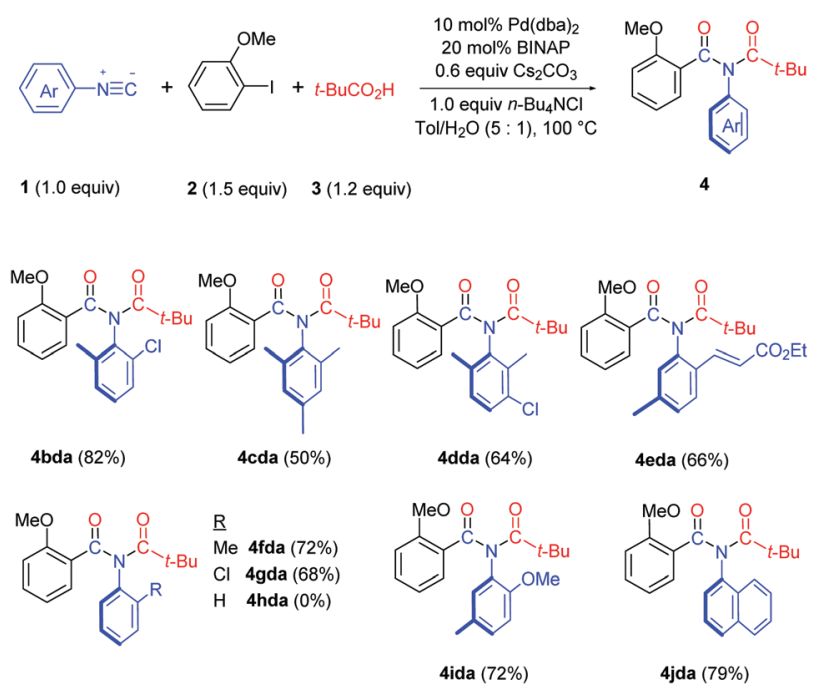

${ }^{a}$ Performed on a $0.20 \mathrm{mmol}$ scale using the standard reaction conditions the same as Table 1 , entry 13. 
Table 4 Scope of carboxylic acids ${ }^{a}$
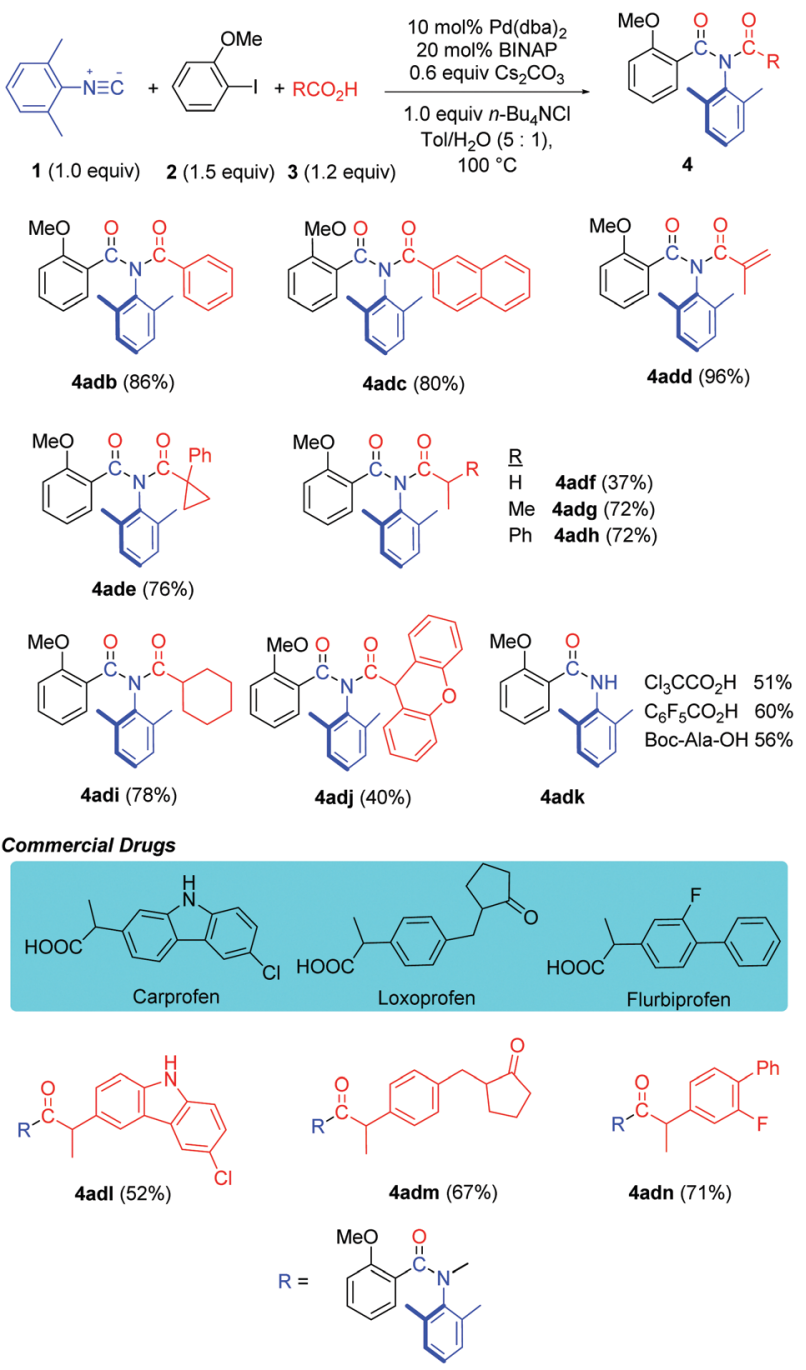

${ }^{a}$ Performed on a $0.20 \mathrm{mmol}$ scale using the standard reaction conditions the same as Table 1, entry 13.

suitable substrate, providing imide 4aia in $71 \%$ yield. We were pleased to find that hetero aromatic halides were compatible with the reaction conditions. Thus, 4-bromoquinoline and 3-iodo-2methoxy pyridine provided the corresponding imide products 4aja and 4aka in good yields.

Both bis-ortho-substituted and mono-ortho-substituted isocyanides afforded good yields of imide products and functional groups such as chloride, methoxy group and acrylate are all well tolerated (Table 3). No imide product 4 hda was obtained when employing isocyanobenzene. This observation demonstrated the importance of steric hindrance of isocyanides, which might promote the reductive elimination of an aryl imidoyl palladium carboxylate intermediate (see Scheme 3). 1-Isocyanonaphthalene afforded imide 4 jda in $79 \%$ yield.

The reaction proceeded very well with various carboxylic acids such as aromatic, vinylic, saturated carbocyclic and benzyl carboxylic acids, providing the corresponding imide products (4adb-adi) in good to excellent yields (Table 4 ). Even very bulky
Table 5 Synthesis of cyclic imides ${ }^{a}$

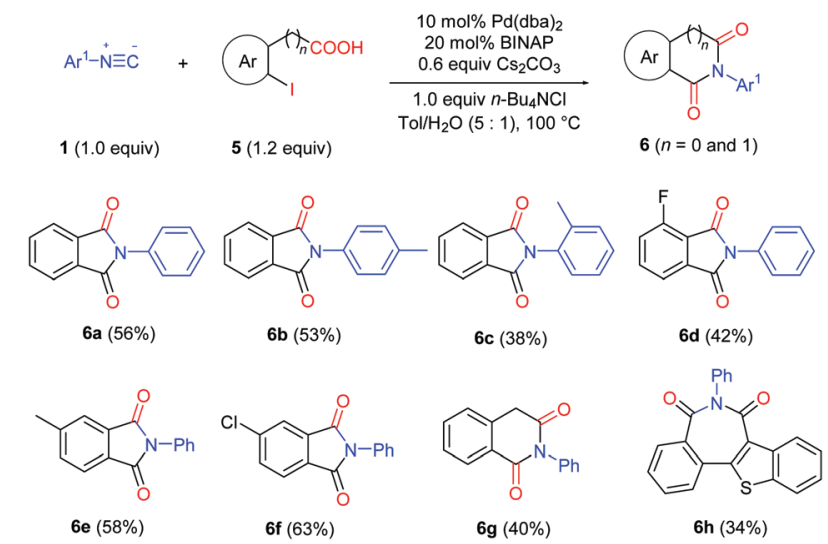

${ }^{a}$ Performed on a $0.20 \mathrm{mmol}$ scale using the standard reaction conditions the same as Table 1 , entry 13.

9H-xanthene-9-carboxylic acid afforded imide 4 adj in a $40 \%$ yield. Interestingly, when employing $\mathrm{Cl}_{3} \mathrm{CCO}_{2} \mathrm{H}, \mathrm{F}_{3} \mathrm{CCO}_{2} \mathrm{H}$ or $N$-protected amino acid Boc-Ala-OH in the reaction, amide 4adk was obtained due to hydrolysis of the corresponding imide products. Gratifyingly, this method has been successfully applied in the late-stage functionalization of drugs Carprofen, Loxoprofen and Flurbiprofen (4adl-adn).

This strategy has also been successfully applied in the synthesis of cyclic imides by using 2-halobenzoic acids (Table 5). Various phthalimides were prepared in moderate to good yields (6a-f). Homophthalimide $\mathbf{6 g}$ and $2 \mathrm{H}$-2-benzazepine-1,3-dione $\mathbf{6 h}$ can also be prepared in useful yields from 2-(2-iodophenyl)acetic acid and 2-(3-iodobenzo[b]thiophen-2-yl)benzoic acid, respectively.

Since the imide products possess $\mathrm{N}$-Ar axial chirality when using asymmetric aryl isocyanides, we performed the reaction using unsymmetrical isocyanides $\mathbf{1 f}$ and $\mathbf{1 k}$ and chiral carboxylic acid (S)-3h (Scheme 2). Slightly enantioenriched products $\mathbf{4 f d h}$ and $\mathbf{4 k \mathbf { k }}$ were obtained. The diastereoselectivity was not improved when employing $(R)$-BINAP as the ligand. ${ }^{13}$

We propose a mechanism for this process as illustrated in Scheme 3 by employing iodobenzene (2a) as an example. $\operatorname{Pd}(0)$ adds oxidatively to iodobenzene to generate the phenylpalladium(II) intermediate I. Isocyanide insertion of this intermediate followed by halide/carboxylate exchange affords imidoylpalladium(II)-carboxylate

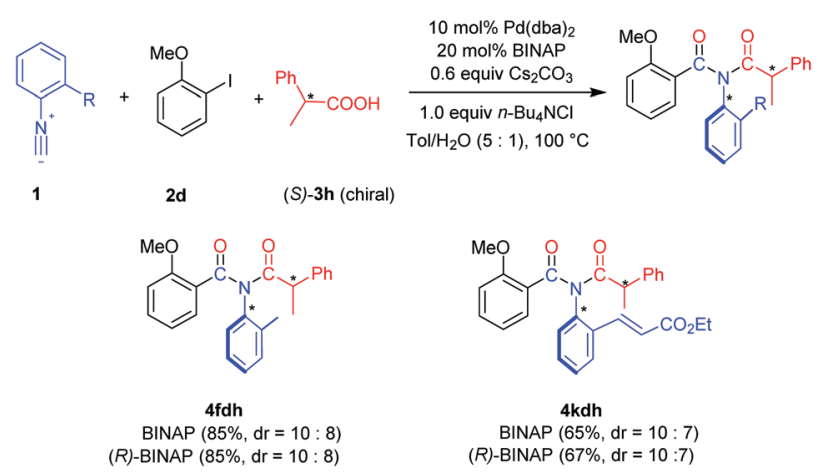

Scheme 2 Diastereoselectivity of palladium-catalyzed Mumm rearrangement. 


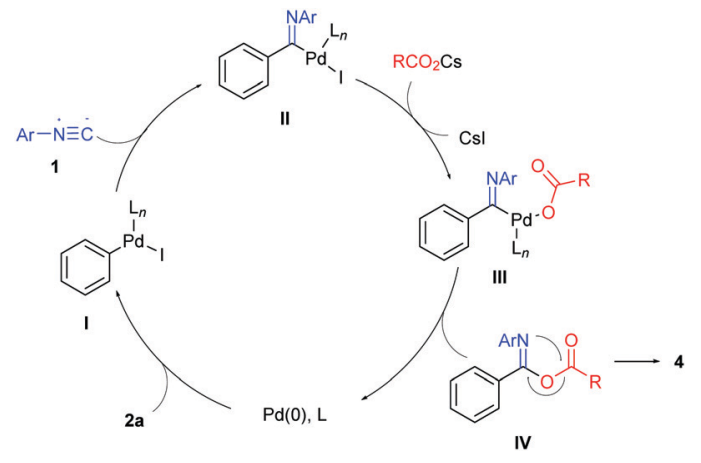

Scheme 3 Proposed mechanism.

complex III, whose reductive elimination leads to isoimide IV and regenerates the $\operatorname{Pd}(0)$ catalyst. $1,3(\mathrm{O}-\mathrm{N})$ acyl transfer of isoimide $\mathbf{~ I V}$ (Mumm rearrangement) produces imide product 4.

In summary, we have developed a general and efficient method to prepare acyclic imides by palladium-catalyzed three-component coupling of aryl halides, isocyanides and carboxylic acids. Key to the success of this approach was controlled isocyanide slow addition and organic/aqueous biphasic conditions. This transition-metal-catalyzed approach features readily available starting materials, atom- and stepeconomy and good functional group compatibility. Additionally, we have also demonstrated expansion of this strategy to the synthesis of cyclic imides.

We are grateful for financial support from Shaanxi University of Science and Technology (BJ15-33).

\section{Conflicts of interest}

There are no conflicts to declare.

\section{Notes and references}

1 (a) T. Pacher, A. Raninger, E. Lorbeer, L. Brecker, P. P.-H. But and H. Greger, J. Nat. Prod., 2010, 73, 1389; (b) A. A. Stierle, D. B. Stierle and B. Patacini, J. Nat. Prod., 2008, 71, 856; (c) G. Ding, L. Jiang, L. Guo, X. Chen, H. Zhang and Y. Che, J. Nat. Prod., 2008, 71, 1861; (d) K. Nakamura, CNS Drug Rev., 2002, 8, 70; (e) S. J. Flatters and G. J. Bennett, Pain, 2004, 109, 150.

2 (a) Y. Hisada and A. Fujinami, Agric. Biol. Chem., 1987, 51, 1547; (b) Z. Cheng, X. Zhu, E. T. Kang and K. G. Neoh, Macromolecules, 2006, 39, 1660.

3 (a) C. Patzelt, A. Pöthig and T. Gulder, Org. Lett., 2016, 18, 3466; (b) S. Takebayashi, J. M. John and S. H. Bergens, J. Am. Chem. Soc., 2010, 132, 12832.

4 (a) O. Mumm, H. Hesse and H. Volquartz, Ber. Dtsch. Chem. Ges., 1915, 48, 379; (b) J. S. P. Schwarz, J. Org. Chem., 1972, 37, 2906.

5 Y. He, Y. Wang, X. Liang, B. Huang, H. Wang and Y.-M. Pan, Org. Lett., 2018, 20, 7117.

6 (a) G. Qiu, C. Chen, L. Yao and J. Wu, Adv. Synth. Catal., 2013, 355, 1579; (b) Z. Hu, D. Liang, J. Zhao, J. Huang and Q. Zhu, Chem. Commun., 2012, 48, 7371; (c) S. Chen, W.-X. Wei, J. Wang, Y. Xia, Y. Shen, X.-X. Wu, H. Jing and Y.-M. Liang, Adv. Synth. Catal., 2017, 359, 3538; (d) J. Peng, L. Liu, Z. Hu, J. Huang and Q. Zhu, Chem. Commun., 2012, 48, 3772.

7 (a) M. Rueping and C. Vila, Org. Lett., 2013, 15, 2092; (b) X. Ye, C. Xie, Y. Pan, L. Han and T. Xie, Org. Lett., 2010, 12, 4240; (c) I. Guerrero, S. S. Marcos and A. Correa, Chem. Commun., 2018, 54, 1627.

8 T. Nanjo, C. Tsukano and Y. Takemoto, Org. Lett., 2012, 14, 4270.

9 Y. Yamamoto, Coord. Chem. Rev., 1980, 32, 193.

10 For selected references using controlled slow addition in Pd-catalyzed reactions, see: (a) R. J. Sullivan, G. P. R. Freure and S. G. Newman, ACS Catal., 2019, 9, 5623; (b) X. Hua, J. Masson-Makdissi, R. J. Sullivan and S. G. Newman, Org. Lett., 2016, 18, 5312; (c) M. Giannerini, M. FañanásMastral and B. L. Feringa, Nat. Chem., 2013, 5, 667; (d) V. Hornillos, M. Giannerini, C. Vila, M. Fañanás-Mastral and B. L. Feringa, Org. Lett., 2013, 15, 5114.

11 CCDC $1962679 \dagger$ contains the supplementary crystallographic data for 4ada.

12 M. Tobisu, S. Imoto, S. Ito and N. Chatani, J. Org. Chem., 2010, $75,4835$.

13 For racemization of chiral imides via $\mathrm{N}$-Ar bond rotation, see: K. Kondo, T. Iida, H. Fujita, T. Suzuki, K. Yamaguchi and Y. Murakami, Tetrahedron, 2000, 56, 8883. 